\title{
Epitaxial growth of (001) and (111) Ni films on MgO substrates
}

\author{
Rosa Alejandra Lukaszew ${ }^{1}$, Vladimir Stoica, Ctirad Uher and Roy Clarke \\ Physics Department, University of Michigan, Ann Arbor \\ ${ }^{1}$ Presently at the Department of Physics and Astronomy, University of Toledo, Ohio.
}

\begin{abstract}
Metal-ceramic interfaces are important in applications as diverse as magnetic storage media and supported catalysts. It is very important to understand how the crystallography and microstructure of metallic films deposited onto ceramic substrates depend on growth and/or annealing conditions so that their physical properties (e.g. magnetic, electronic, etc.) can be tailored for specific applications. To this end, we have studied the epitaxial growth and annealing of (001) and (111) Ni films MBE grown on $\mathrm{MgO}$ substrates, where we have observed the evolution of the surface using correlated insitu RHEED (reflection high-energy-electron diffraction) and STM (scanning tunneling microscopy) measurements.
\end{abstract}

\section{INTRODUCTION}

We have previously shown that epitaxial single-crystal magnetic thin films may be used in spin-dependent tunneling applications [1]. In general, the magnetic properties, particularly the anisotropy [2], of epitaxial thin films are dominated by the crystallographic structure of the metal/substrate interface as well as the surface quality. In addition, for spin-dependent tunneling devices, the roughness at the surface must be very small in order to ensure the integrity of the subsequent deposition of ultra-thin, pinhole-free insulating layers. Thus, we have considered the growth of magnetic films on $\mathrm{MgO}$ substrates, which can be prepared with very smooth surfaces [3].

Theoretical studies have indicated that for $\mathrm{Ni}$ films grown on $\mathrm{MgO}$ substrates, $\mathrm{Ni}$ is expected to strongly interact with $\mathrm{MgO}$ [4]. Various researchers have studied the orientation of $\mathrm{Ni}$ films on $\mathrm{MgO}$ substrates under various growth conditions [5], and some reports indicate that $\mathrm{Ni}$ may form an epitaxial relationship with $\mathrm{Ni}[001] / \mathrm{MgO}[001]$ and $\mathrm{Ni}(010) / / \mathrm{MgO}(010)$ for films deposited using dc sputtering on $\mathrm{MgO}$ substrates held at $100^{\circ} \mathrm{C}[6]$.

There are also reports on epitaxial growth of $f c c$ metals on surfaces with hexagonal surface symmetry such as $\mathrm{MgO}$ (111) [7]. Sandström et al. [8] have shown that at growth temperatures between $300^{\circ} \mathrm{C}$ and $400^{\circ} \mathrm{C}$ it is possible to grow smooth $\langle 111\rangle$ oriented single domain epitaxial films on $\mathrm{MgO}$ substrates, utilizing dc magnetron sputtering in an ultra high vacuum (UHV) chamber. In the following, we present our studies on the molecular beam epitaxy (MBE) growth/annealing and in-situ surface structural characterization of single domain Ni films grown on (001) and (111) oriented $\mathrm{MgO}$ substrates.

\section{EXPERIMENTAL}

The Ni films were grown in an MBE VG $80 \mathrm{M}$ system with a background pressure $<5 \times 10^{-11}$ torr. Ni was evaporated from a $99.999 \%$ pure source. The deposition 
rate was $0.5 \AA / \mathrm{sec}$. The substrates used in the experiment were $0.5 \mathrm{~mm}$ thick, $1 \mathrm{x} 1 \mathrm{~cm}^{2}$ pre-polished $\mathrm{MgO}(001)$ and (111) oriented single crystals, which were heat-treated in UHV at $800^{\circ} \mathrm{C}$ for $1 \mathrm{hr}$. The combination of flat polished substrates and the UHV heating cycle to allow the surface layers to regain crystalline order has been proven to permit growth of single crystal metal films [6] as well as exhibiting sharp reflection high-energy electron diffraction (RHEED) from the $\mathrm{MgO}$ surface.

$E x$-situ atomic force microscopy (AFM) characterization of the annealed surfaces showed smooth surfaces with a root mean square (rms) surface roughness of $0.2 \mathrm{~nm}$ for the $(001)$ oriented substrates and $0.5 \mathrm{~nm}$ for the (111) oriented ones. Prior to initiating the growth, the substrate temperature was lowered to the appropriate deposition temperature for metal growth $\left[\mathrm{T} \cong 100{ }^{\circ} \mathrm{C}\right.$ for $(001)$ and $\mathrm{T} \cong 300{ }^{\circ} \mathrm{C}$ for $(111)$ oriented Ni films]. Heat transfer was by direct radiation between the heater and $\mathrm{MgO}$ substrate. The RHEED patterns were recorded continuously during deposition and during subsequent annealing of the films [9]. The surface morphology of the as-deposited and annealed films was determined in-situ with scanning tunneling microscopy [(STM) RHK model STM100].

\section{DISCUSSION}

\section{(001) oriented Ni films}

The RHEED pattern of the heat-treated (001) MgO substrates showed long streaks characteristic of a smooth, single-domain surface. Sharp Kikuchi lines indicated long-range lateral coherence. The substrate temperature was lowered to $100^{\circ} \mathrm{C}$ for deposition, which was determined to be optimal for single-domain growth. The Ni film's final thickness was $50 \mathrm{~nm}$. The Ni RHEED pattern evolved from wide and diffuse streaks at the beginning of the growth into sharper and spotty streaks indicating threedimensional growth [Fig. 1(a)]. The RHEED pattern for a $50 \mathrm{~nm}$ thick film indicated single crystal structure for all azimuthal orientations. The mounded quality of the surface was corroborated with in-situ STM. [Fig. 3(a)] The rms surface roughness of the asgrown films was $0.5 \mathrm{~nm}$. In order to further smoothen the surface, the films were annealed in UHV at $573 \mathrm{~K}(\sim 1 / 3$ of the Ni melting temperature) for several hours.
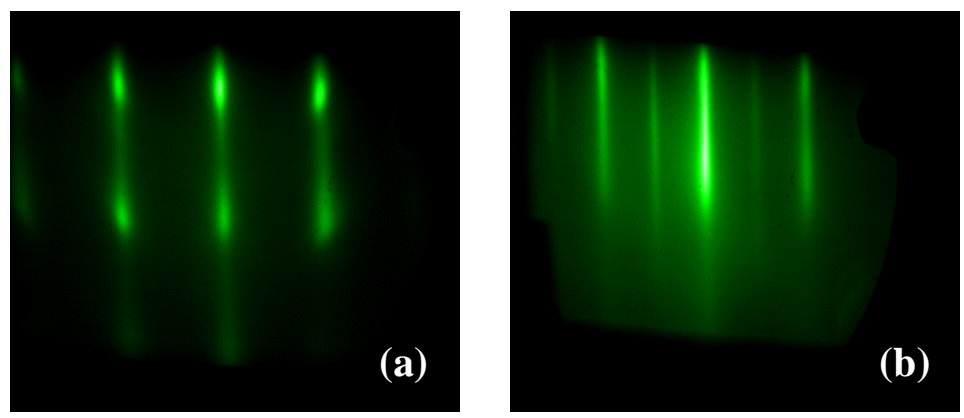

Figure 1. (a) RHEED pattern of the "as grown" (001) $\mathrm{Ni}$ film. (b) RHEED pattern of the same film after annealing. 


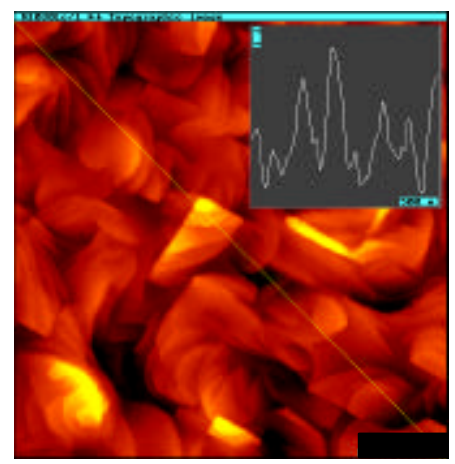

Figure 2. STM image of the (001) Ni surface after annealing in $\mathrm{UHV}$ at $573 \mathrm{~K}$. The scale bar corresponds to $50 \mathrm{~nm}$.

Inset: line-scan along the diagonal of the STM image, showing the resultant roughness. The bar on the lower right corner corresponds to $50 \mathrm{~nm}$. The bar on the upper left corner corresponds to $0.1 \mathrm{~nm}$.
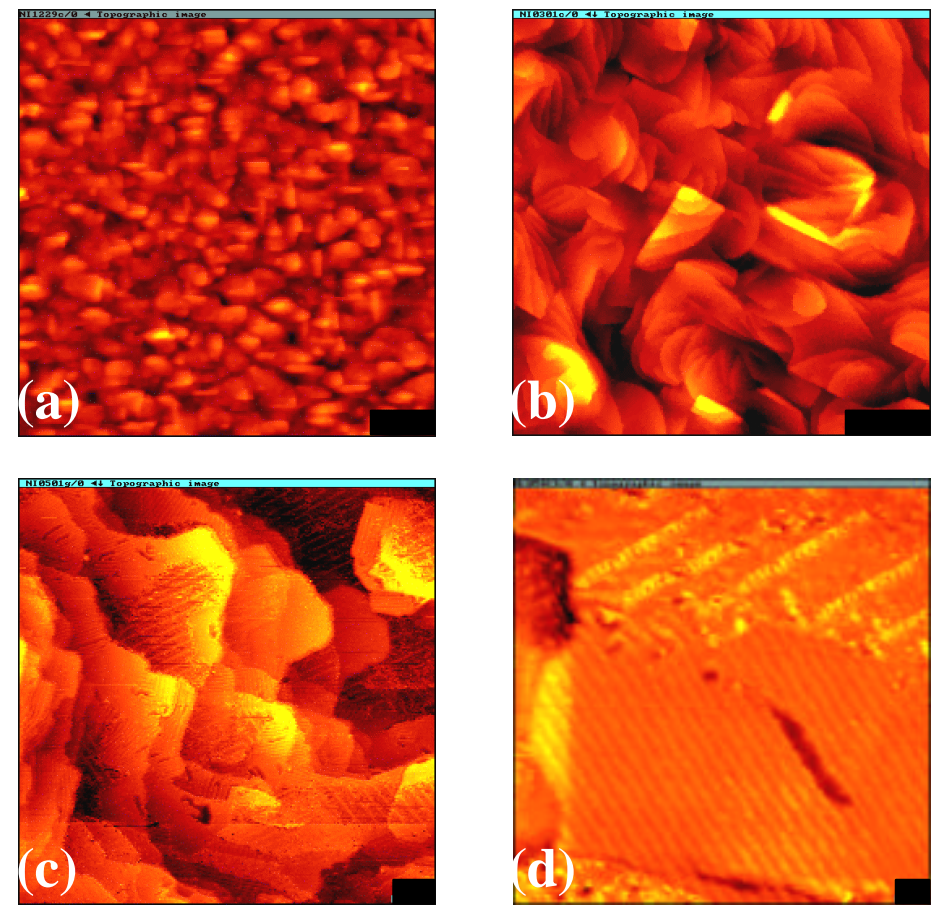

Figure 3. (a) STM image of the "as grown" (001) Ni film. (b) STM image of the same film after annealing. The scale bar at the bottom right corner corresponds to $50 \mathrm{~nm}$. In (c) and (d) the scale bar corresponds to $10 \mathrm{~nm}$ and $2 \mathrm{~nm}$, respectively. 
The sharpening of the RHEED pattern during annealing indicated a better crystalline quality as well as smoothening of the surface. It also showed the presence of half-order streaks [Fig. 1(b)] typical of a (2x1) surface reconstruction. STM imaging of the annealed surface shows that the annealing was dominated by "turbulent step flow" due to the presence of defects, mainly screw dislocations [Fig. 2 and Fig. 3 (b)]. The rms surface roughness of the annealed films was $0.2 \mathrm{~nm}$.

Higher magnification of the annealed surface showed periodic reconstructions [Fig 3 (c and d)] with two distinct periodicities. A longer period $(2.1 \mathrm{~nm})$ and a shorter one $(0.7 \mathrm{~nm})$, the latter consistent with the $(2 \times 1)$ reconstruction observed with RHEED.

In order to understand these reconstructions we first considered the possible effect of strain. The lattice misfit between $\mathrm{MgO}$ and $\mathrm{Ni}$ is $16 \%$. However, it has been postulated [6] that an in-plane super-cell matching (commensuration) between the film and substrate with $\mathrm{a}_{\mathrm{o}}(\mathrm{Ni}) \times 6=2.0446 \mathrm{~nm}$ and $\mathrm{a}_{\mathrm{o}}(\mathrm{MgO})$ × $5=2.1066 \mathrm{~nm}$ will reduce the misfit to $\sim 0.8 \%$. The critical thickness needed to relieve such a small strain may be quite large. Still, some authors [10] claim that super-cell matching itself cannot give rise to the formation of single crystalline Ni layers, as it has been shown that in other cases interfacial periodic reconstructions can exist that allow for single crystal growth. Our observations support this. Annealing the films relaxed the surface and evidenced a reconstruction with periodicity related to the size of the postulated super-cell (i.e. 2.1 $\mathrm{nm})$.

The (2x1) missing row reconstruction has been observed before, in Mo films grown on $\mathrm{MgO}$, and ascribed to the presence of oxygen at the surface [11]. Assuming the same possibility here, it has a minor effect because ex-situ magnetic characterization of the samples indicates that they are ferromagnetic and not dominated by oxide formation (Fig.4). Presuming oxygen contamination of the surface, the oxygen atoms may be positioned in three-fold coordinated sites on the Ni fcc lattice, which are shifted off the top position on the top Ni layer towards the underlying Ni layer. Thus, the top Ni atoms are surrounded by oxygen, which prevents the formation of a complete top Ni layer, hence the missing-row type surface reconstruction.

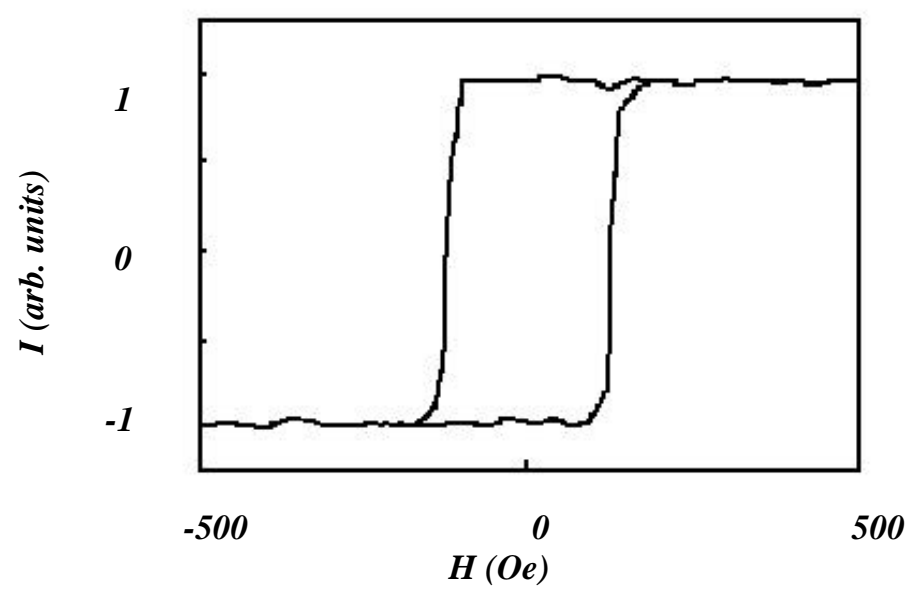

Figure 4. MOKE hysteresis loop for the annealed (001) Ni film. The external field is applied along the $\langle 110>$ crystallographic direction. 
The Ni films were grown via evaporation from an ultra-pure Ni source and in a $<5$ $\mathrm{x} 10^{-11}$ torr environment, suggesting then that oxygen may evolve from the $\mathrm{MgO}$ substrate and segregate to the surface during the annealing stage.

\section{(111) oriented Ni films}

After the UHV thermal pre-treatment, the (111) MgO substrate temperature was lowered to $300^{\circ} \mathrm{C}$ for deposition, the optimal temperature for single crystal growth [8]. The RHEED pattern indicated that the first two monolayers of growth are strained with a lattice parameter close to that of $\mathrm{MgO}$. The growth proceeds relaxed afterwards with $\mathrm{Ni}$ lattice parameter close to the bulk value. [Fig. 5(a)] Thus, for this case, as the strained layers are energetically unfavorable, the rapid relaxation may be achieved via formation of a large number of defects and dislocations.

The RHEED pattern confirmed single domain film during the growth and it is also the same as that of the substrate, indicating that $\mathrm{Ni}$ grows with the same stacking sequence [i.e. (111) oriented $f c c$ ] as the underlying $\mathrm{MgO}$ substrate. The substrate temperature ensures enough energy and mobility to the subsequent adatoms so that they can find the lowest energy position resulting in a single-domain structure. The STM image of a $25 \mathrm{~nm}$ film [Fig. 5(b)] shows large islands with central vacancies associated with dislocations. The rms surface roughness is $0.3 \mathrm{~nm}$.
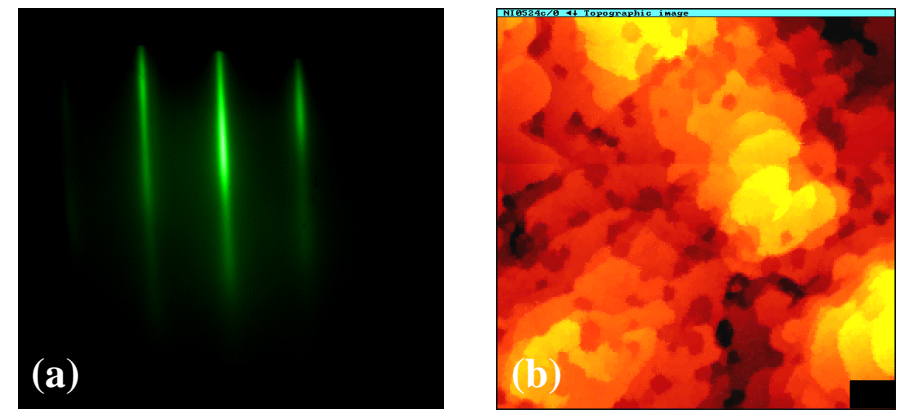

Figure 5. (a) RHEED pattern of the "as grown" (111) Ni film. (b) STM image of the surface of the same film. The bar at the bottom right corner corresponds to $10 \mathrm{~nm}$.

\section{CONCLUSIONS}

We have been able to grow epitaxial single-domain smooth $\mathrm{Ni}$ films on $\mathrm{MgO}$ substrates in both (001) and (111) orientations using MBE. STM imaging of the (001) annealed Ni surface indicates two different types of periodic reconstruction. A longer period reconstruction may be associated with super-cell formation at the interface in order to reduce the lattice misfit with the substrate. The shorter period "missing row" 
(2x1) reconstruction, also observed with RHEED, might be understood assuming the presence of oxygen segregated from the substrate to the surface during annealing. The ferromagnetic character of the (001) Ni films and not the oxide dominates the magnetic properties of the films.

No surface reconstruction was observed for (111) Ni films grown on $\mathrm{MgO}$ substrates under similar conditions. In addition, for the (111) case, strain relaxation occurred at the initial stages of the growth via formation of lattice defects and dislocations.

Both types of films have adequate surface roughness to be used in spin-dependent structures. A complete magnetic characterization of the films is currently in progress.

\section{REFERENCES}

1. R. A. Lukaszew, Y. Sheng, C. Uher, and R. Clarke, Appl. Phys. Lett. 75, 1941 (1999).

2. K. B. Hathaway and G. Prinz, Phys. Rev. Let. 47, 1761 (1981); R. A. Lukaszew, E. Smith, R. Naik, C. Uher, R. Clarke, submitted to Phys. Rev. Lett.

3. S. S. Perry, P. B. Merrill, Surf. Sci. 383, 268 (1997).

4. G. Pacchioni, N. Rösch, J. Chem. Phy. 104, 7329 (1996).

5. H. Qiu, H. Nakai, M. Hashimoto, G. Safran, M. Adamik, P.B. Barna, E. Yagi, J. Vac. Sci. Technol. A 12, 2855 (1994); J. P. McCaffrey, E. B. Svedberg, J. R. Phillips, L. D. Madsen, J. Cryst. Growth 200, 498 (1999).

6. E. B. Svedberg, P. Sandström, J. -E. Sundgren, J. E. Greene, L. D. Madsen, Surf. Sci. 429, 206 (1999).

7. P. Haibach, J. Koble, M. Huth, H. Adrian, Thin Solid Films 336, 168 (1998).

8. $\quad$ P. Sandström, E. B. Svedberg, J. Birch, J. -E. Sundgren, Surf. Sci. 437, L767 (1999).

9. D. Barlet, C. W. Snyder, B. G. Orr, and R. Clarke, Sci. Instrum. 62, 1263 (1991); data acquisition using KSA400, k-Space. Assoc. Inc., Ann Arbor, MI 48109.

10. A. Zur, T. C. McGill, J. Appl. Phys. 55, 278 (1984).

11. H. Xu, K. Y. S. Ng, Surf. Sci. 355, L305 (1996). 